\title{
Osteoblastic cell response on high-rough titanium coatings by cold spray
}

\author{
A.M.Vilardell $^{1 *}$, N. Cinca ${ }^{1}$, N.Garcia-Giralt ${ }^{2}$, S.Dosta $^{l}$, I.G.Cano ${ }^{l}$, X.Nogués $^{2}$, J. M. Guilemany $^{l}$ \\ ${ }^{1}$ Centre de Projecció Tèrmica (CPT). Dpt. Ciència dels Materials i Enginyeria Metal.lúrgica. Universitat de \\ Barcelona Martí i Franquès 1, 08028 Barcelona, Spain. \\ ${ }^{2}$ IMIM (Institut Hospital del Mar d'Investigacions Mèdiques), RETICEF, Doctor Aiguader 88, 08003 Barcelona, \\ Spain
}

*Corresponding author: amvilardell.research@gmail.com

\begin{abstract}
Highly rough and porous commercially pure titanium coatings have been directly produced for first time by the cold spray technology, which is a promising technology in front of the vacuum plasma spray for oxygen sensitive materials. The wettability properties as well as the biocompatibility evaluation have been compared to a simply sand blasted Ti6Al4V alloy substrate. Surface topographies were analysed using confocal microscopy. Next, osteoblast morphology (Phalloidin staining), proliferation (MTS assay), and differentiation (alkaline phosphatase activity) were examined along 1, 7 and 14 days of cell culture on the different surfaces. Finally, mineralization by alizarin red staining was quantified at 28 days of cell culture.
\end{abstract}

The contact angle values showed an increased hydrophilic behaviour on the as-sprayed surface with a good correlation to the biological response. A higher cell viability, proliferation and differentiation were obtained for highly rough commercial pure titanium coatings in comparison with sand blasted substrates. Cell morphology was similar in all coatings tested; at 14 days both samples showed extended filopodia. A higher amount of calcium-rich deposits was detected on highly rough surfaces. In summary, in-vitro results showed an increase of biological properties when surface roughness increases.

KEYWORDS: Highly-rough surface; titanium coatings; cold spray; in-vitro; joint prosthesis; 


\section{INTRODUCTION}

Titanium and titanium alloys require a surface treatment in order to become bioactive and thus, being osteoproductive materials [1]. In this way, the formation of a fibrous soft tissue capsule can be avoided, enabling better biomechanical fixation [2]. Therefore, the implant surface is a key role which has been addressed by many authors and still attracts the interest day by day with the aim to enhance osseointegration and promote faster fixation. The causes of prosthetic loosening can be as a result of foreign-body reaction to wear particles and early prosthetic instability, as well as bone resorption and subsequent clinical loosing and inadequate interlock [3,4].

The titanium surface modification can involve topography as well as compositional changes, thus modifying the wettability performance and the final biological properties [5,6,7]. Mekayarajjananonth actually reported a number of studies supporting the relationship among surface factors, including surface preparation, roughness, surface energy, contact angle values, and cell adhesion to biomaterial surfaces (Fig.1) [8].

It is reported that implant surface roughness plays a role in determining phenotypic expression of cells in vivo [9] as well as cell adhesion $(\leq 24 \mathrm{~h})$ depended on the available surface area [10]. Many authors have compared several of the existing titanium surface treatments for the assessment of in vitro performance $[11,12,13]$. Le Guehennec et al. [11] compared the biological response of several surfaces with different properties (mirror-polished, alumina grit blasted, biphasic calcium phosphate ceramic grit blasted and a commercially available implant surface (SLA). It was shown that osteoblastic cell attachment, spread, and proliferation were initially (4 days of culture) more rapid on smooth surfaces than on rough surfaces $(\mathrm{Ra}=2.5 \mu \mathrm{m})$; however it turns out at 15 days of culture with a higher proliferation in the rough surfaces. Osteoblast differentiation was higher in rough surfaces along the cell culture. Moreover, smooth $\mathrm{Ti}$ and plastic substrates with similar hydrophilicity and roughness show different viability, probably due to surface charge and composition that affect protein adsorption. Also, surfaces with comparable roughness showed different cell adhesion and proliferation because of their hydrophilicities.

The perfect surface roughness is the one that combines micro-/submicro-scale roughnesses. Rolando et al. [14] developed a simple oxidation treatment for generating controlled nanoscale topographies on Ti surfaces, while retaining the starting micro-/submicro-scale roughness. The introduction of such nanoscale structures in combination with micro-/submicro-scale roughness improves osteoblast differentiation and local factor production which, in turn, 
indicates the potential for improved implant osseointegration in vivo [15]. During this last decade, there has been enough interest in the study of anodized surfaces for inducing nanotexturing surfaces [16].

A new direction of material development has been taken in the research of rough/porous coating surfaces for improving biological properties of hip prosthesis [17]. Rough and porous surfaces have emerged as versatile biomaterials for enhancing fixation to bone being suitable for hip and knee replacements [18]. Actual prosthetic femoral implants are even designed with different distinct zones, each zone having its own roughened surface creating a tripartite differential porosity [19].

Up to date, there is a wide range of surface modification treatments and it is worth noting that the concept of smooth and rough surfaces needs to be clarified. Wennerberg et al. [20] actually reviewed several surface topography parameters related to several of these treatments; they state the difficulty on comparing the findings of the many existing works due to different characterization techniques or reported parameters used for the study. Also, when the surface topography is changed, the surface chemistry or physics may change simultaneously. Furthermore, when the surface microtopography is changed, the nanotopography of the same surface usually changes too, even without being planned by the investigator. Therefore, caution must be considered when dealing with all these issues.

Plasma Spraying (PS) is considered a surface modification technique at the micrometer level [1] and is a wellestablished process for commercial prosthesis that have proved long-term fixation [21,22]. PS surfaces are often used in combination with other modifications such as blasting or etching [9,23]. However, for titanium spraying usually vacuum plasma spraying is used, which increases the cost of the process. Current high rough CP-Ti coatings are produced by VPS [24] $(\mathrm{Ra}=74 \mu \mathrm{m})$, and they are already used in orthopaedics with good results in-vitro [24] and invivo $[25,26]$ results, but with a higher. Even many works based on the revision after clinical use can be found in the literature $[27,28,29]$.

As an alternative, Cold Spray (CS) technology allows the obtaining of oxide-free coatings at room pressure conditions at a lower cost [30]. Solid feedstock powders, (normally in the range of 5 to $50 \mu \mathrm{m}$ ), are accelerated in a supersonic gas at velocities up to $1100 \mathrm{~m} / \mathrm{s}$. The propelled particles impact on the substrate and undergo plastic deformation and adhere to the substrate. As a solid-state process, it is ideal to spray temperature-sensitive materials to avoid possible oxidation in comparison with the conventional thermal spray techniques. Dense titanium coatings have been pursued potentially for aeronautical and aerospace industry [30]. However, porous coating could be quite 
useful in biomedical applications. Based on the plastic deformation capability of the feedstock powder, results in top surface coating morphologies where possible existing small protuberances of the individual particles are well maintained in the as-sprayed surface. Therefore, it leads to micro+nanotopography without the need of posterior treatments.

Some authors have previously obtained CS commercial pure $\mathrm{Ti}$ (CP-Ti) coatings for biomedical applications [31,32,33,34]. The use of porogen materials has enabled the production of suitable porosities for bone ingrowth promotion but both large roughness and porosity are difficult to obtain. The use of coarse feedstock in cold gas spray can provide both features at the same time [35]. Previously, such coatings have proved to accomplish the mechanical standard specifications for prostheses applications. Here we present an in vitro study of this highly rough CS CP-Ti coating in comparison with a sand blasted (SB) microrough surface in order to biologically characterize the influence of both topography surfaces, with emphasis on the morphological features of the CS process.

\section{MATERIALS AND METHODS}

\subsection{Sample preparation and surface characterization}

Ti6Al4V alloy specimens were sand-blasted using an alumina \#24 mesh. Samples of the same type were used for CS of titanium particles by means of a CGS KINETICS® 4000 (Cold Gas Technology, Ampfing, Germany) operated with nitrogen as the propellant gas [35]. A CP-Ti grade 2 powder of a particle size between $90-150 \mu \mathrm{m}$ and irregular shape (MBN Nanomaterialia SpA) was used as feedstock .7x7x2 $\mathrm{mm}^{3}$ specimens were used for in-vitro tests. The samples were examined by Scanning Electron Microscopy (SEM) using a JEOL 5310 operated at $20 \mathrm{kV}$ and equipped with an Energy Dispersive Spectroscopy (EDS) for microanalysis. Specific surface and 2D surface topography measurements were carried out with Confocal Microscopy Leica DCM3D. The (micro)roughness and waviness values of the samples were extracted from the global profile with a Gaussian filter 0,25mm and according to ISO 4287. A home-made water contact angle goniometer with ImageJ software program has been used to the measurement of the contact angles, performing static measurements in sessile drop mode at 20s. These measurements were obtained by depositing 3-10 drops of $2 \mu \mathrm{m}$ of MilliQ water onto the surface of the samples.

\subsection{Cell culture}


Human osteoblastic cells (hOB) have been obtained from knee trabecular bone after prosthesis replacement following the protocol described by Nacher et al. [36]. The study has been approved by the of Parc de Salut Mar Ethics Committee. Briefly, trabecular bone was dissected into 1-2 mm pieces that were washed in phosphatebuffered solution (PBS) and placed into a $15 \mathrm{~cm}$ diameter Petri dish containing $15 \mathrm{ml}$ Dulbecco's modified Eagle's medium (DMEM) supplemented with 10\% FBS, penicillin (100 UI/ml), streptomycin (100 UI/mL), ascorbic acid (100 mg/ml) (Invitrogen) and fungisone (0.4\%) (Gibco). The explants were incubated at $37{ }^{\circ} \mathrm{C}$ in a humidified atmosphere of $5 \% \mathrm{CO} 2$, changing the medium once a week until cell confluence. Finally, cells were changed into new $75 \mathrm{~cm}^{2}$ flasks until the suitable number has been reached. A maximum of a third subculture has been used in the experiments. For materials testing, samples were overnight sterilized in ethanol $70^{\circ}$, washed in PBS and placed on a 48-well polystyrene culture plate (Nunc A/S). Each material was seeded with 100.000 cells and cultured with DMEM supplemented with 10\% FBS and ascorbic acid; for mineralization assays $\beta$-glycerophosphate (5mM) was also added. Before each tests, seeded materials were removed from the original well and put into another one in order to ensure the results obtained are provided only by the cells attached on the studied coatings, and not by the cells remaining on the plate surface. Samples were tested at 1, 7 and 14 days of cell culture, except for mineralization assay that was performed at 28 days.

\subsection{Cell viability and proliferation assays}

Cell proliferation has been tested using the MTS assay CellTiter 96® AQueous One Solution Cell Proliferation assay (Promega) according to manufacturer's protocol. $50 \mu \mathrm{l}$ of MTS were added in each sample cultured with $250 \mu \mathrm{l}$ of supplemented medium, incubating for 3 hours and then recording the absorbance at 490nm.

LIVE/DEAD Viability/Cytotoxicity assay Kit for Mammalian Cells (Invitrogen) was performed in order to characterize cell viability, attachment and distribution. It discriminates live from dead cells by simultaneously staining with green-fluorescent calcein-AM (life cells) and red-fluorescent ethidium homodimer-1 (dead cells). Live/Dead assay was performed by adding $300 \mu \mathrm{l}$ of a solution at $4 \mu \mathrm{M}$ EthD-1 and $2 \mu \mathrm{M}$ of calcein $\mathrm{AM}$ in Phosphate-buffered saline (PBS), per sample and incubated for 30-45 min at room temperature. Then, surfaces have been observed with a Confocal TCS SP5 Upright from Leica Microsystems and the images were processed with Fiji software.

\subsection{Alkaline phosphatase assay}


Osteoblastic cell differentiation levels were evaluated through Alkaline Phosphatase (ALP) activity, using the Abcam's Alkaline Phosphatase Assay Kit. This assay uses p-nitrophenyl phosphate (pNPP) as a phosphatase substrate which turns yellow when dephosphorylated (it turns to p-nitrophenol) by ALP. The resulting absorbance was measured using a scanning multi-well spectrophotometer, at $405 \mathrm{~nm}$.

\subsection{Alizarin red stainning}

The cells attached to the samples were washed with PBS and fixed with $10 \%$ formamide for 10 min. Samples were washed with PBS and stained with $300 \mu \mathrm{l}$ of 40mM/1 Alizarin red solution, pH 4.2 (Sigma-Aldrich) per well at room temperature for $10 \mathrm{~min}$ under gentle shaking. The unincorporated dye was removed and samples were washed carefully with PBS to remove excess stain. Then, mineralization was quantified by dissolving the precipitated Alizarin red with $10 \%$ cetylpyridinium chloride solution at room temperature during 30 min on gentle shaking. 100 $\mu \mathrm{l}$ of the stained solutions were quantified at $550 \mathrm{~nm}$.

\subsection{Cell morphology}

Phalloidin-Tetramethylrhodamine B isothiocyanate (Sigma-Aldrich) staining were used for determining the structure of the cytoskeleton of cells seeded onto the materials. Cells were cleaned with PBS several times and fixed 10 minutes in $3.7 \%$ formaldehyde (Probus) solution in PBS. Then, cells were washed extensively again in PBS and permeabilized with $0.1 \%$ TRITON® X-100 (Sigma-Aldrich) in PBS 5 minutes and gently rinsed with PBS. After that, cells were washed again in PBS and stained with a 50mg/ml fluorescent phalloidin and 4',6-diamidino phenylindole (DAPI) (0,2 mg/ml) (Sigma-Aldrich)in PBS (protected from the light) 40 min at room temperature. Cells were observed with a Leica Confocal TCS SP5 Upright.

\subsection{Statistical analysis}

Statistical analyses were performed by Mann-Whitney U test for group comparisons in the SPSS v.12.0 for Windows. All analyses were two-tailed, and p-values $<0.05$ were considered significant. Tests were carried out three times in order to ensure reproducibility. Each test contained two replicas of each sample and was tested together positive and negative controls. Results were normalized by the SB samples within each experiment and each time in order to see differences among the materials within the same time. 


\section{RESULTS}

\subsection{Surface characterization of titanium samples}

The morphological characterization of titanium surfaces shows the distinct topographical features of both the SB and CS surfaces (Fig.2); figures 2a and b show the sand blasted surface morphology, which is characteristic of the eroding effect of angular shaped alumina particles when the material is displaced forming rims or lips. On the other hand, figure 2c shows the large irregular shaped titanium particles deposited by CS. At higher magnification, a nanotopography morphology can be distinguished, which is dependent on the surface asperities of the feedstock that can be preserved after spraying or further features that can appear as result of particles deformation upon impact (Fig.2d).

Figure 3 shows the 2D surface roughness and waviness profiles. SB produces a less rough and less complex surface than the CS CP-Ti coating. Both samples showed microroughness with a distance between peaks on the order of tens of microns. Even so, SB surfaces manifest less deep groves in comparison with CS CP-Ti coatings. As CS CP-Ti coatings were produced by coarse particles, a larger amplitude in the waviness profile could be observed in comparison with the SB surface. The combination between microroughness along waviness profile leads to a high increase of surface area. 3D parameters such as Sdar (developed surface) and Spar (Projected surface) were measured to reflect that enlargement. SB samples increased $x 4.5$ its surface area while the CS CP-Ti coating increased x11 in comparison with a \#240 grinded Ti6Al4V surface. Table 1 summarizes all those roughness profiles values.

Figure 4 shows the contact angle of MilliQ water onto both surfaces. On SB surfaces-showed hydrophilic behaviour. The contact angle values for SB surfaces and CP-Ti coatings were of $33 \pm 2^{\circ}$ and $26 \pm 10^{\circ}$ respectively, in comparison with $73 \pm 2^{\circ}$ (result not shown) from a $\# 240$ grinded Ti surface.

\subsection{Osteoblast viability}

The Live/Dead assay showed good cell viability on both surfaces (Fig. 5). Most of the cells were alive after 14 days of cell culture. No significant dead was found in any of both specimens. In correlation with the Live/Dead assay, cells seeded on CS CP-Ti coatings showed more MTS activity than cells on SB surfaces at all days of culture, especially at 7 days of culture (Fig. 6). 


\subsection{Osteoblast function}

The ALP activity detected in cells on CS CP-Ti coatings is higher in comparison with SB surfaces at all times of cell culture, especially at 14 days in which differences are significant (Fig. 7). Moreover, cells seeded on CP-Ti coatings also showed more mineralization levels than SB surface after 28 days of cell culture (Fig. 8).

\subsection{Morphological aspects of osteoblast}

Figure 9 shows cell morphology micrographs of osteoblast cells at 1,7 and 14 days of culture. The actin cytoskeleton was marked by Rhodamine (fluorescent red colour) and the nucleus by Dapi (blue colour). On both surfaces, cells showed a flattened and spread cytoskeleton with long cytoplasmic extensions.

\section{DISCUSSION}

Through the present work it has been observed that porous and highly rough titanium coatings can be obtained by means of Cold Gas Spraying of coarse feedstock particles rather than spherical finer particles as employed in most of other studies. Large sized irregular particles in the range between 63 and $106 \mu \mathrm{m}$ were also used to produce aluminium coatings [37], but in that case the purpose was not to obtain porous coatings. Coarse particles result in lower spraying velocities and larger gaps to be filled among the deposited particles. The CGS technology is a quite complex process where also powder and substrate mechanical, physical and chemical properties need to be considered, which can be revised elsewhere [38]. The proper metallurgical bonding among particles requires the disruption of native oxide films on the individual particles which seems to be accomplished at the observed contact interfaces.

The spraying of coarse particles also provides certain porosity and little deformation upon impact, which could be beneficial for osseointegration and cell attachment. Actually, such surface texturization rather than microroughness can determine cell adhesion and proliferation [39].

Concerning the use of titanium surface treatments for prosthesis, a lot of studies are focused on the biological properties induced by micro- and nano- rough surfaces, although the surface roughness criteria is not very clear yet. Some of the commercial specification/requirements report roughness (Ra) values $>15 \mu \mathrm{m}$ for Tibiocoat ${ }^{[}[40]$, or $(\mathrm{Rt})$ $>100 \mu \mathrm{m}$ Ti-Grip ${ }^{\circledR}$ and Ti-Pore ${ }^{\circledR}$ by VPS [41]. The obtained CS CP-T coatings have Rt values $>200 \mu \mathrm{m}$ and 
reached a global profile value of $\mathrm{Ra} 40 \mu \mathrm{m}$ (with a surface microroughness of $\mathrm{Ra} \sim 12 \mu \mathrm{m}$ and a surface waviness of Wa $11 \mu \mathrm{m}$ ) by using a particle size mostly above $100 \mu \mathrm{m}$. The benefit from obtaining a higher surface area will lead to an increase of surface for cell attachment, thus favouring osseointegration. The increase of surface roughness showed an increase of hydrophilicity. The contact values obtained from a grinded Ti surface $\left(-73^{\circ}\right)$, decreased to $33^{\circ}$ after SB treatment and $\sim 26^{\circ}$ after spraying the coating, with the increase of surface roughness $(\sim 4 \mu \mathrm{m}$ for SB surface and $\sim 40 \mu \mathrm{m}$ for CP-Ti coating).

The effect of surface energy, water contact angle or wettability has to be considered as it affects protein adsorption, cell adhesion, proliferation and osteoblastic differentiation. Hence, implant wettability can play a key role for the protein adsorption and consequently, for the cell adhesion. It is usually reported that biomaterial surfaces with moderate hydrophilicity provide better cell growth and higher biocompatibility [42]. Surface wettability is considered to increase production of osteoblastic factors and bone formation as well as roughness [43]. Rough VPS titanium coatings have also shown to be hydrophilic. Biolin Scientific reported comparison study between rough VPS titanium coatings and different titanium surfaces was carried out according to their roughness and wettability [44]. It was observed that the highest surface roughness $(\mathrm{Sa}=27.8 \mu \mathrm{m})$ and hydrophilicity $\left(37^{\circ}\right)$ was obtained by rough VPS Ti coating, thus making it a possible candidate as a bone implant surface. In addition, it is also reported that the combination of micro/nano-texturization yields dramatically smaller distilled water contact angles $\left(16.22^{\circ}\right)$ $[45]$.

The surface topography and wettability provided by CS CP-Ti coatings provide better cell response in comparison with SB surfaces. Cells seeded on both surfaces showed good cell attachment onto low and higher surface roughness. Cells showed flattened morphology, displaying extended cytoskeleton on surface specimens. However, cell viability and proliferation was detected to be higher for CP-Ti coating at all days of cell culture tested, and especially significant at 7 days. Irregular highly rough surfaces, as well higher wettability provided by CS technique, have a direct effect on osteoblast attachment and subsequent proliferation. Boyan et al. [46] observed that cell proliferation increased with the increase of surface topography too. The combination of higher surface microroughness and higher surface waviness suggest a better cell anchoring. Cells are stimulated by different levels of surface roughness, as long as those levels are not greater than the cell dimensions, in which would not enhance cell response [24]. As well, the higher wettability of CP-Ti coatings may help to that behaviour. On the other hand, ultra-high rough VPS Ti 
coatings $(\mathrm{Ra}=74 \mu \mathrm{m})$ have been tested in comparison with low-roughness Ti coatings $(\mathrm{Ra}=18 \mu \mathrm{m}$ and $\mathrm{Ra}=40 \mu \mathrm{m})$. Results show that they provided a good biological response. Even though, at least in vitro, they behaved similarly to the coatings already used in orthopaedic [47]. However, ultra-high values of roughness are not correctly seen by cells [48].

In addition, the rapid cell attachment and proliferation of cells onto CP-Ti coatings contributes to detect higher values of ALP compared to SB surfaces. Hence, the largest cell number in CP-Ti coatings at all times correlates with the higher ALP and mineralization levels detected in each corresponding time. It is already reported that osteoblastlike cells adhere more readily and showed to be more differentiated on rougher surfaces, with regards to morphology, extracellular matrix production and ALP activity [43] Mariscal-Muñoz et al. [15] observed an increase of cell differentiation as well as of cell mineralization with the increase of surface roughness between two different titanium grade 4 surfaces, one polished with a $\mathrm{Ra}=0.3 \mu \mathrm{m}$, and a second one roughened by laser irradiation with and $\mathrm{Ra}=10.6 \mu \mathrm{m}$. Mendonça et al. [49] reported an increase of mineralization with an increase of surface roughness. Rough surface topography positively modulated expression of genes related to collagen biosynthesis and crosslinking in adherent cells associated with an increased deposition of collagen-rich matrix.

\section{CONCLUSIONS}

The biological feasibility of highly rough titanium coatings produced by CS was tested, showing that the CS technique provides differential surface features compared to conventional PS since this does not involve particle melting and it is just rather based on direct particle bonding when plastic deformation is produced upon impact, avoiding at the same time, any oxidation of the titanium. The use of coarse feedstock particles allowed the obtaining of highly rough surface as well as a microtexturization given by particle feedstock morphologies.

In vitro results of such developed coatings compared to a simply SB surface showed an increase of biological properties when surface roughness was increased. Cell morphology was very similar on SB surface and CS CP-Ti, showing good spreading and flattening for all observation times. Higher cell viability and cell proliferation were observed for CS coatings; they also showed higher ALP and mineralization levels in comparison with SB surfaces. 
The CP-Ti coating produced by CS seems to be a good alternative to produce coatings with very good mechanical and biological properties for joint cementless prostheses application in a cost-effective way and using a friendlyenvironment technology compared to others, in comparison with VPS.

\section{ACKNOWLEDGMENTS}

The authors want to thank the Spanish MINECO for financial support through project MAT2013-46755-R and the Generalitat de Catalunya for the project 2014 SGR 1558, and University of Barcelona for the award of a scholarship that has helped the development of this research. This work was also supported by the Red Temática de Investigación Cooperativa en Envejecimiento y Fragilidad (RETICEF; RD12/0043/0022), the Centro de Investigación Biomédica en Red de Fragilidad y Envejecimiento Saludable (CIBERFES; CB16/10/00245) and FEDER funds.

\section{REFERENCES}

[1] Bauer S, Schmuki P, von der Mark K, Park J. Engineering biocompatible implant surfaces. Progress in Materials Science. 2013;58:261-326.

[2] Elias CN, Lima JHC, Valiev R, Meyers MA. Biomedical applications of titanium and its alloys. JOM. 2008;60:46-9.

[3] Pramanik S, Agarwal AK, Rai KN. Chronology of Total Hip Joint Replacement and Materials Development. Artif Organs. 2005;19:15-26.

[4] Mjöberg B. Theories of wear and loosening in hip prostheses. Wear-induced loosening vs loosening-induced wear--a review. Acta Orthop Scand. 1994;65:361-71.

[5] Rupp F, Scheideler L, Olshanska N, de Wild M, Wieland M, Geis-Gerstorfer J. Enhancing surface free energy and hydrophilicity through chemical modification of microstructured titanium implant surfaces. J Biomed Mater Res A. $2006 ; 76$ A:323-34. 
[6] Rupp F, Scheideler L, Rehbein D, Axmann D, Geis-Gerstorfer J. Roughness induced dynamic changes of wettability of acid etched titanium implant modifications. Biomater. 2004;25:1429-38.

[7] Gnedenkov SV, Sinebryukhov SL, Egorkin VS, Mashtalyar DV, Alpysbaeva DA, Boinovich LB. Wetting and electrochemical properties of hydrophobic and superhydrophobic coatings on titanium. Colloids Surf A: Physicochem Eng Asp. 2011;383:61-6.

[8] Mekayarajjananonth T, Winkler S. Contact angle measurement on dental implant biomaterials. J Oral Implantol. $1999 ; 25: 230-6$.

[9] Martin JY, Schwartz Z, Hummert TW, Schraub DM, Simpson J, Lankford J, Dean DD, Cochran DL, Boyan BD. Effect of titanium surface roughness on proliferation, differentiation, and protein synthesis of human osteoblast-like cells (MG63). J Biomed Mater Res. 1995;29:389-401.

[10] Rosales-Leal JI, Rodríguez-Valverde MA, Mazzaglia G, Ramón-Torregrosa PJ, Díaz-Rodríguez L, GarcíaMartínez O, Vallecillo-Capilla M, Ruiz C, Cabrerizo-Vílchez MA. Effect of roughness, wettability and morphology of engineered titanium surfaces on osteoblast-like cell adhesion. Colloids Surf A: Physicochem Eng Asp. 2010;365:222-9.

[11] Le Guehennec L, Lopez-Heredia M-A, Enkel B, Weiss P, Amouriq Y, Layrolle P. Osteoblastic cell behaviour on different titanium implant surfaces. Acta Biomater. 2008;4:535-43.

[12] Wall I, Donos N, Carlqvist K, Jones F, Brett P. Modified titanium surfaces promote accelerated osteogenic differentiation of mesenchymal stromal cells in vitro. Bone. 2009;45:17-26.

[13] Degasne I, Baslé MF, Demais V, Huré G, Lesourd M, Grolleau B, Mercier L, Chappard D. Effects of Roughness, Fibronectin and Vitronectin on Attachment, Spreading, and Proliferation of Human Osteoblast-Like Cells (Saos-2) on Titanium Surfaces. Calcified Tissue Int. 1999;64:499-507.

[14] Gittens RA, McLachlan T, Olivares-Navarrete R, Cai Y, Berner S, Tannenbaum R, Schwartz Z, Sandhage KH, Boyan BD. The effects of combined micron-/submicron-scale surface roughness and nanoscale features on cell proliferation and differentiation. Biomaterials. 2011;32:3395-403. 
[15] Mariscal-Muñoz E, Costa CAS, Tavares HS, Bianchi J, Hebling J, Machado JPB, Lerner UH, Souza PPC. Osteoblast differentiation is enhanced by a nano-to-micro hybrid titanium surface created by $\mathrm{Yb}$ :YAG laser irradiation. Clin Oral Investig. 2016;20:503-11.

[16] Oh S, Daraio C, Chen L-H, Pisanic TR, Fiñones RR, Jin S. Significantly accelerated osteoblast cell growth on aligned TiO2 nanotubes. J Biomed Mater Res A. 2006;78A:97-103.

[17] Le Guéhennec L, Soueidan A, Layrolle P, Amouriq Y. Surface treatments of titanium dental implants for rapid osseointegration. Dental Mate. 2007;23:844-54.

[18] Muth J, Poggie M, Kulesha G, Michael Meneghini R. Novel Highly Porous Metal Technology in Artificial Hip and Knee Replacement: Processing Methodologies and Clinical Applications. JOM. 2013;65:318-25.

[19] I. Murray, Differential porosity prosthetic hip system, Google Patents, 2007. https://www.google.ch/patents/US20070043446.

[20] Wennerberg A, Albrektsson T. Effects of titanium surface topography on bone integration: a systematic review. Clin Oral Implants Res. 2009;20:172-84.

[21] Lombardi AV, Berend KR, Mallory TH, Skeels MD, Adams JB. Survivorship of 2000 Tapered Titanium Porous Plasma-sprayed Femoral Components. Clin Orthop Relat Res. 2009;467:146-54.

[22] Ellison B, Berend KR, Lombardi AV, Mallory TH. Tapered Titanium Porous Plasma-Sprayed Femoral Component in Patients Aged 40 Years and Younger. J Arthroplasty. 2006;21:32-7.

[23] Taba Júnior M, Novaes AB, Souza SL, Grisi MF, Palioto DB, Pardini LC. Radiographic evaluation of dental implants with different surface treatments: an experimental study in dogs. Implant Dent. 2003;12:252-8.

[24] Borsari V, Giavaresi G, Fini M, Torricelli P, Tschon M, Chiesa R, Chiusoli L, Salito A, Volpert A, Giardino R. Comparative in vitro study on a ultra-high roughness and dense titanium coating. Biomater. 2005;26:4948-55.

[25] Frauchiger VM, Eitel F,Tommasini R, Jaeggi C, Wippich T, Jaeggi S. An Open-Porous Titanium Coating for Advanced Osseointegration. 55th Annu Mtg Orthop Res Soc. 
[26] Endres S, Wilke M, Knöll P, Frank H, Kratz M, Wilke A. Correlation of in vitro and in vivo results of vacuum plasma sprayed titanium implants with different surface topography. J MateR Sci Mater Med. 2008;19:1117-25.

[27] Braem A, Chaudhari A, Vivan Cardoso M, Schrooten, Duyck J, Vleugels J, Peri- and intra-implant bone response to microporous Ti coatings with surface modification. Acta biomater. 2014;10:989-995

[28] Salemyr M, Muren O, Eisler T, Bodén H, Chammout G, Stark A, Sköldenberg O. Porous titanium construct cup compared to porous coated titanium cup in total hip arthroplasty. A randomised controlled trial. Int Orthop. 2015; $39(5): 823-32$

[29] Lakstein D1, Backstein D, Safir O, Kosashvili Y, Gross AE. Revision Total Hip Arthroplasty with a Porouscoated Modular Stem: 5 to 10 Years Followup. Clin Orthop Relat Res. 2010; 468(5):1310-1315.

[30] Moridi A, Hassani-Gangaraj SM, Guagliano M, Dao M. Cold spray coating: review of material systems and future perspectives. Surface Engineering. 2014;30:369-95.

[31] Sun J, Han Y, Cui K. Innovative fabrication of porous titanium coating on titanium by cold spraying and vacuum sintering. Materials Letters. 2008;62:3623-5.

[32] Qiu D, Zhang M, Grøndahl L. A novel composite porous coating approach for bioactive titanium-based orthopedic implants. J Biomed Mater Res. 2013;101A:862-72.

[33] Vilardell AM, Cinca N, Concustell A, Dosta S, Cano IG, Guilemany JM. Cold spray as an emerging technology for biocompatible and antibacterial coatings: state of art. J Mate Sci. 2015;50:4441-62.

[34] Dosta S, Cinca N, Garcia J, Guilemany JM, Ti Deposition onto Ti6Al4V Alloy by Cold-Gas Spraying in Medical Engineering ESB2009. European Conference on Biomaterials. Ref 1212. Publicación en CD.1 pag. Lausanne. Switzerland. (2009).

[35] Guilemany JM, Cinca N, Dosta S, Cano IG. Intelectual Property: Feasibility of cold gas spraying to produce high roughness high porous titanium coatings for metallic prosthesis, Ref Number 1870 - . Universitat de Barcelona. Legal Deposit: 17/10/2014, Spain, 2014 
[36] Nàcher M, Aubia J, Serrano S, Mariñoso ML, Hernández J, Bosch J, Díez A, Puig JM, Lloveras J. Effect of cyclosporine A on normal human osteoblasts in vitro. Bone Miner. 1994;26:231-43.

[37] Van Steenkiste TS, Smith JR, Teets RE, Aluminum coatings via kinetic spray with relatively large power particles. Surf. Coat. Technol. 2002; 154:237-252.

[38] Champagne Victor K (2007) The cold spray materials deposition process: fundamentals and applications. Woodhead, Cambridge

[39] Rosales-Leal JI, Rodríguez-Valverde MA, Mazzaglia G, Ramón-Torregrosa PJ, Díaz-Rodríguez L, GarcíaMartínez O, Vallecillo-Capilla M, Ruiz C, Cabrerizo-Vílchez MA. Effect of roughness, wettability and morphology of engineered titanium surfaces on osteoblast-like cell adhesion. Colloids Surf A: Physicochem Eng Asp. 2010;365:222-9.

[40] http://www.tlsmedical.com/

[41]http://www.eurocoating.it/plasma_spray_coatings/vacuum_plasma_spray_titanium_coatings/default.aspx

[42] Janssen MI, van Leeuwen MBM, van Kooten TG, de Vries J, Dijkhuizen L, Wösten H a. B. Promotion of fibroblast activity by coating with hydrophobins in the beta-sheet end state. Biomater. 2004;25:2731-9.

[43] Hotchkiss KM, Reddy GB, Hyzy SL, Schwartz Z, Boyan BD, Olivares-Navarrete R. Titanium surface characteristics, including topography and wettability, alter macrophage activation. Acta Biomater. 2016;31:425-34.

[44]http://www.biolinscientific.com/zafepress.php?url=\%2Fpdf\%2FAttension\%2FApplication\%20Notes\%2FAT_A N_17_roughbio.pdf

[45] Zhao L, Mei S, Chu PK, Zhang Y, Wu Z. The influence of hierarchical hybrid micro/nano-textured titanium surface with titania nanotubes on osteoblast functions. Biomater. 2010;31:5072-82.

[46] Boyan BD, Bonewald LF, Paschalis EP, Lohmann CH, Rosser J, Cochran DL, Dean DD, Schwartz Z, Boskey AL. Osteoblast-Mediated Mineral Deposition in Culture is Dependent on Surface Microtopography. Calcified Tissue Int. 2002;71:519-29. 
[47] Borsari V, Giavaresi G, Fini M, Torricelli P, Tschon M, Chiesa R. Comparative in vitro study on a ultra-high roughness and dense titanium coating. Biomater. 2005; 26:4948-4955

[48] Borsari V, Giavaresi G, Fini M, Torricelli P, Salito A, Chiesa R, Chiusoli L, Volpert A, Rimondini L, Giardino R. Physical Characteriztion of Different-Roughness Tianium Surfaces, With and Without Hydroxyapatite Coating, and Their Effect on Human Osteoblast-Like Cells. J Biomed Mater Res. 2005; 75B:359-368

[49] Mendonça DBS, Miguez PA, Mendonça GA, Yamauchi M, Aragão FJL, Cooper LF, Titanium surface topography affects collagen biosynthesis of adherent cells, Bone. 2011; 49:463-472.

\section{FIGURE CAPTIONS}

Fig.1. Relationship among surface properties with cell adhesion

Fig.2. SEM micrographs at different magnifications of a-b) SB surfaces and c-d) CS CP-Ti coatings

Fig.3. Surface topography of SB surfaces and CS CP-Ti coatings

Fig.4. Contact angles of MillQ water droplets on a) SB surfaces and b) CS CP-Ti coatings

Fig.5. Live/Dead assay at 1, 7 and 14 days of cell culture (from left to right) onto a,b,c) SB surfaces and d,e,f) CS CP-Ti coatings $(n=3)$

Fig.6. MTS assay at 1, 7 and 14 days of cell culture of SB surfaces and CS CP-Ti coatings $(\mathrm{n}=3$; *p-values $<0.05)$

Fig.7. ALP assay at 1, 7 and 14 days of cell culture of SB surfaces and CS CP-Ti coatings $(\mathrm{n}=3$; *p-values $<0.05)$

Fig.8. Alizarin Red assay at 28 days of cell culture of SB surfaces and CS CP-Ti coatings $(n=3 ; * p-v a l u e s<0.05)$

Fig.9. Phalloidin stainning at 1, 7 and 14 days of cell culture (from left to right) onto a,b,c) SB surfaces and d,e,f) CS CP-Ti coatings $(n=3)$ 